\title{
Stickstoffbilanz bei Patienten nach laparoskopischer Cholezystektomie
}

\author{
Nitrogen Balance in Patients after Laparoscopic Cholecystectomy
}

\author{
Z. Kopański1,2, Małgorzata Schlegel-Zawadzka³, A. Cienciała1', B. Witkowska1, K. Czajecki1, Z. Kustra1
}

\begin{abstract}
Zusammenfassung: Bei 251 Patienten mit laparoskopischer Cholezystektomie und bei 230 Patienten mit laparotomischer Cholezystektomie (Kontrollgruppe) wurde postoperativ die Intensität der katabolen Phase untersucht. Die Untersuchung umfaßte die Bestimmung der Stickstoffbilanz über 3 Tage durch Messung der Ausscheidung von Harnstoff-N im Urin. Die Stickstoffbilanz war bei allen untersuchten Patienten negativ. Bei Patienten mit laparoskopischer Cholezystektomie war der Wert der negativen Stickstoffbilanz im Durchschnitt 3 mal geringer als bei Patienten nach traditioneller Cholezystektomie.
\end{abstract}

Schlüsselwörter: Cholezystektomie, Laparoskopische; Harnstoff/Urin; Postoperative Komplikationen/Stoffwechsel; Stickstoff/Stoffwechsel.

Summary: The intensity of catabolic phase in the post-operative period was evaluated in 251 patients who underwent laparoscopic cholecystectomy and in a control group of 230 patients who underwent cholecystectomy by laparotomy. The balance of nitrogen was assessed over 3 days by measurement of urea- $\mathrm{N}$ excreted in the urine. Nitrogen-balance was negative in all operated patients. Compared to the patients with cholecystectomy by classical laparotomy, the loss in nitrogen was threefold lower in the patients after cholecystectomy by laparotomy.

Keywords: Cholecystectomy, Laparoscopic; Nitrogen/metabolism; Postoperative Complications/metabolism; Urea/urine.

\footnotetext{
1 Chirurgische Klinik und Poliklinik Norstand: Oberst Doz. Dr. med. habil. A. Cienciała) des Militärkrankenhauses, Kraków, Polen 2 Korrespondenzadresse: Dr. Z. Kopański, Chirurgische Klinik und Poliklinik, Militärkrankenhaus, Wroclawska-Straße 3, PL-30-901 Kraków, Polen

${ }^{3}$ Lehrstuhl für Bromatologie und Ernährung der Jagiellonischen Universität, Kraków, Polen (Norstand: Prof. Dr. habil. Z. Zachwieja)

Eingegangen: 9. Jan. 1996 / Angenommen: 31. Mai 1996
}

Seit der mehr als 110 Jahre zurückliegenden erstmaSligen Gallenblasenentfernung durch Langenbuch im Jahre 1882 [1] war die Cholezystektomie mittels der Laparotomie das Verfahren der Wahl bei der Behandlung der unkomplizierten Cholezystolithiasis. Der entscheidende Wendepunkt für diese Behandlung erfolgte erst im März 1987, als Mouret [2] die Laparoskopie zur Gallenblasenentfernung anwandte. Mit der erfolgreichen Anwendung dieser Methode begann eine außerordentlich dynamische Entwicklung dieser Operationstechnik. Auch in Polen wurde diese Technik schnell akzeptiert und erfolgreich eingeführt. Trotz der hohen instrumentellen Kosten wird heute bereits in zahlreichen polnischen Kliniken die laparoskopische Cholezystektomie durchgeführt. Auch bei Operationseingriffen an anderen Organen im Abdominal- und Thoraxbereich, wie z. B. bei der Appendektomie, der segmentalen Enterektomie. der Leberresektion, den Hernien-, Magen- oder Lungenoperationen, wird immer häufiger die Laparoskopie angewandt [2-19].

Nach laparoskopischen Operationseingriffen beobachtet man wesentlich kürzere Genesungszeiten als bei Patienten, die sich den traditionellen Operationseingriffen unterzogen haben. Eine objektive Bewertung der „Reaktion des Organismus auf das Operationstrauma", das der laparoskopische Eingriff verursacht, fand bis jetzt kaum statt. Dies veranlaßte uns, eigene Untersuchungen hinsichtlich der Stickstoffbilanz bei Patienten nach laparoskopischer Cholezystektomie duchzuführen.

\section{Patienten und Methodik -}

In der Gruppe von 1839 Patienten, die von Januar 1994 bis Januar 1996 in unserer Klinik wegen der Cholezystolithiasis operiert wurden, wurde in 613 Fällen die Stickstoffbilanz prospektiv untersucht. Aus der letztgenannten Gruppe wurden zur weiteren Analyse 481 Patienten ausgewählt. Diese Patientengruppe war charakterisiert durch eine kurze (in der Regel ein- bis zweitätige) Vorbereitungszeit zur Operation, keine Begleitkrankheiten (z. B. Diabetes), die einen Einfluß

J Lab Med 1996; 20 (9) : 487-489 
Tabelle 1 Tägliche Zufuhr von Stickstoff, Elektrolyten, Energieträgern und Wasser bei Patienten nach Cholecystektomie während der 3tägigen Untersuchungsperiode ${ }^{1}$

\begin{tabular}{llllll}
\hline Patientengruppe & $\begin{array}{l}\mathrm{N} \\
\text { (g) }\end{array}$ & $\begin{array}{l}\mathrm{K+} \\
\text { (mmol) }\end{array}$ & $\begin{array}{l}\mathrm{Na}+ \\
\text { (mmol) }\end{array}$ & $\begin{array}{l}\text { Energie } \\
\text { (kJ) }\end{array}$ & $\begin{array}{l}\mathrm{H}_{2} \mathrm{O} \\
\text { (I) }\end{array}$ \\
\hline $\begin{array}{l}\text { Gruppe 1 } \\
\text { (laparoskopische }\end{array}$ & 0 & 29,4 & 122,2 & 836 & 1,4 \\
$\begin{array}{l}\text { Cholecystektomie) } \\
\text { Gruppe 2 }\end{array}$ & 0 & 32,5 & 126,5 & 836 & 1,4 \\
$\begin{array}{l}\text { (Cholecystektomie } \\
\text { durch Laparotomie) }\end{array}$ & & & & & \\
\hline 1Angaben pro m² Körperoberfläche & & \\
\hline
\end{tabular}

auf die Stickstoffbilanz ausüben könnten, und eine vergleichbar unkomplizierte postoperative Phase (z. B. cine ähnlich kurze Drainierung aus der Operationsivunde). Unterschiedlich war nur die Technik der Gallenblasenentfernung. Aus dieser Basis wurden die untersuchten Patienten in zwei Gruppen unterteilt:

Gruppe 1: 251 Patienten (18 Männer und 233 Frauen) im Alter von 20 bis 65 Jahren (Durchschnittsalter 40.5 Jahre), welche einer laparoskopischen Cholezystektomie unterzogen wurden. Die mittlere Operationsdauer belief sich auf 42 Minuten.

Gruppe 2 (Kontrollgruppe): 230 Patienten (13 Männer und 217 Frauen) im Alter von 21 bis $63 \mathrm{Jah}$ ren (Durchschnittsalter 41,3 Jahre), bei denen die Gallenblasenentfernung mittels der Laparotomie durchgeführt wurde. Die mittlere Operationsdauer belief sich auf 50 Minuten.

Im analysierten zweijährigen Zeitraum wurden beide Operationstechniken in unserer Klinik mit einer ähnlichen Häufigkeit verwendet.

Während der Untersuchungen blieben die Patienten unter Standardbedingungen; die Stickstoffzufuhr wurde vereinheitlicht (Tabelle 1).

Die Intensität der katabolen Phase wurde mittels der Stickstoffbilanz bewertet, die im Laufe der ersten 3 Tage nach dem Eingriff untersucht wurde. Die Stickstoffausscheidung wurde aufgrund der Messung des Harnstoff-N im 24h-Sammelurin bestimmt, gemäß der folgenden Formel:

Stickstoffverlust pro Tag $(\mathrm{g} / \mathrm{d})=$

$$
\frac{\text { U-Harnstoff-N }(\mathrm{g} / \mathrm{d})}{0,75}+2
$$

Die Messung der Harnstoffkonzentration im Urin erfolgte mit dem Analysator RA-1000 der Fa. Technicon unter Verwendung der vom Hersteller empfohlenen Reagenzien.

Vor dem Beginn der Bilanzuntersuchungen wurde bei allen Patienten eine akute und chronische Niereninsuffizienz ausgeschlossen. Die Bilanzergebnisse wurden Quadratmeter Körperfläche umgerechnet.

Die statistische Auswertung der Ergebnisse wurde mittels des Programmpakets SAS rel. 6.03 für IBM PC durchgeführt. Für beide Gruppen der Patienten wurde jewcils der Mittelwert $\overline{\mathbf{x}}$ und die Standardabweichung (SD) berechnet. Die Hypothese über die Normalvertcilung wurde mittcls des von Shapiro-Wilk angegebenen Testes W (SAS Procedures Guide, rel. 6.113) verifizicrt. Dic Berechnungen wurden mittels der Prozedur „UNIVARIANTE“ realisiert. Zur Bewertung der Differenzen zwischen den Mittelwerten wurde der I-Tcst nach Student angewandt. Als Signifikanzniveau wurde $p<0.05$ festgelegl.

\section{Ergebnisse}

Die Untersuchungsergebnisse sind in der Tabelle $2 \mathrm{zu}-$ sammengefaßt. Bemerkenswert ist die statistisch signifikante Differenz zwischen den Mittelwerten der 3-Tage-Stickstoffbilanz bei Patienten nach laparoskopischer und laparotomischer Cholezystektomie. Bei Patienten, die der Cholezystektomie mittels der Laparoskopie unterzogen wurden, ist die mittlere negative Stickstoffbilanz 3 mal geringer als bei Patienten nach traditioneller Cholezystektomie.

\section{Diskussion}

Jede „Verletzung" des Organismus löst eine Reaktion aus, die erstens die Erhaltung des möglichst konstanten Volumens der extrazellulären Flüssigkeit und zweitens die Deckung des in diesen Zuständen zunehmenden Energiebedarfs des Organismus zum Ziel hat: insbesondere wird die Gluconeogenese erhöht. Die Hauptquelle der Glukose für den Organismus ist nach dem Aufbrauchen der Glukagonbestände und nach der Einstellung der exogenen Zufuhr - sein eigenes Protein. In der katabolen Phase des posttraumatischen Zustands treten Änderungen sowohl des Proteinabbaus als auch der Proteinsynthese auf, wobei der Abbau die Synthese übersteigt.

Das Maß der Intensität dieses Prozesses ist die negative Stickstoffbilanz. Sie wird durch Menge des insgesamt ausgeschiedenen und zugeführten Stickstoffes bestimmt. Die Stickstoffausscheidung verläuft in $90 \%$ über den Urin. Daher spiegelt der Harnstoffstickstoff

Tabelle 2 Stickstoffbilanz bei Patienten nach Cholecystektomie während der 3tägigen Untersuchungsperiode 1

\begin{tabular}{|c|c|c|c|c|}
\hline Patientèngruppe & $\begin{array}{l}\text { Ausge- } \\
\text { schiedene } \\
\text { Menge } \\
\bar{x}\end{array}$ & $\begin{array}{l}\text { Zufuhr } \\
\bar{x}\end{array}$ & Bilanz & SD \\
\hline $\begin{array}{l}\text { Gruppe 1 } \\
\text { (laparoskopische } \\
\text { Cholecystektomie) } \\
\text { Gruppe } 2 \\
\text { (Cholecystektomie } \\
\text { durch Laparotomie) }\end{array}$ & 3,72 & 0 & $\begin{array}{l}-3,72^{2} \\
-10,81\end{array}$ & $\begin{array}{l}2,59 \\
4,61\end{array}$ \\
\hline
\end{tabular}


- nach der Meinung vieler Untersucher - den Proteinkatabolismus wider [20,21]. Aus unseren Untersuchungen ergab sich, daß bei allen Patienten, die einer Cholezystektomie unterzogen wurden, eine negative Stickstoffbilanz auftrat. Ihr Ausmaß hing statistisch signifikant von der angewandten Operationstechnik ab. Nach laparoskopischen Eingriffen war die negative Stickstoffbilanz 3 mal kleiner als nach traditioneller Cholezystektomie.

Zur weitverbreiteten Meinung über die laparoskopischen Eingriffe, daß sie sich durch kürzere Hospitalisationszeit $[3,4]$ und günstigen kosmetischen Effekt [22] kennzeichnen, kann man demnach hinzufügen, daß sie ein kleineres Operationstrauma im Vergleich zu den traditionellen chirurgischen. Methoden auslösen.

\section{Literatur}

1. Langebuch C. Ein Fall von Extirpation der Gallenblase wegen chronischer Cholelithiasis. Heilung. Berl Klin Wochenschr 1882;19: 725-801.

2. Cuschleri A, Dubois F, Moutel J, Mouret Ph, Becker H, Buess G. Trede M, Troidl $H$. The European experience with laparoscopic cholecystectomy. Am J Surg 1991; 161:385-8.

3. Bailey RW, Flowers JL, Graham SM, Zucker KA. Combined laparoscopic cholecystectomy and selective vagotomy. Surg Laparosc Endosc 1991:1:45-9.

4. Bendawid R. New techniques in hernia repair. World J Surg 19S9:13:522-7.

5. Cadiére GB. Himpens J. Bruyns J. Laparoscopic proximal gastric vagotomy. End Surg 1994;2:105-8.
6. Corrbitt JD. Laparoscopic herniography. Surg Laparosc Endosc 1990;1:23-6.

7. Crocc E, Azzola M. Russo R. Golia M. Angelini S. Olimi S. Laparoscopic liver tumour resection with the argon beam. End Surg $1994 ; 2: 186-8$.

8. Cucto J, Weber A, Serrano F. Laparoscopic treatment of perforated duodenal ulcer. Surg Laparose Endosc 1993; 3:216-8.

9. Dubois E. Vagotomies - laparoscopic or thoracoscupic approach. End Surg 1994:2:100-4.

10. Ger R, Mishrick A, Hurwitz J, Romero J, Oddsen R. Management of groin hernias by laparoscopy. World J Surg 1993;17:46-50.

11. Goetz F, Pier A, Bacher C. Die laparoskopische Appendekımie. Chirurg 1991:62:253-8.

12. Katkhouda N. Heimbucher J. Mouiel J. Laparoscopic posterior vagotomy and anterior sero-myotomy. End Surg 1994:2:95-9.

13. Katkhouda N. Mouiel J. Laparoscopic treatment in inguinal hernias. End Surg 1993;1:193-7.

14. Kavic MS. Laparoscopic repair of ruptured duodenal peptic ulcer: a case report. J Laparosc Surg 1993;3:41-5.

15. Kum CK, Ngoi SS, Goh PMY, Tekant Y. Isaac JR. Randomized controlled trial comparing laparoscopy and open appendicectomy. Br J Surg 1993;80:1599-1600.

16. Lanreneau RJ, Hazalrigg SR, Ferson PF. Johnson JA, Nawarawong W, Herla DB, Dowling RD. Thoracoscopic resection of 85 pulmonary lesions. Ann Thorac Surg 1992:54:415-420.

17. Lointier PH. Lautard M, Massoni C. Ferrier C. Dapoingny M. Laparoscopically assisted subtotal colectomy. J Laparoendoscop Sur 1993;3:439-53.

18. Querleu D. Laparoscopically assisted radical vaginal hysterectomy. Ginecol Oncol 1993:51:248-54.

19. Schreiber JH. Results of outpatient laparoscopic appendectomy in women. Endoscopy 1994;26:292-8.

20. Montgomery R, Dryer RL. Conivay TW, Spector AA. Biochemistry. St. Louis (USA): CV Mosby Company, 1993.

21. Murray RK, Granner DK, Mayes PA, Rodwell VW. Harper's Biochemistry. Warszawa (Polen): PZWL, 1994.

22. Rudowski W. Chirurgia laparoskopowa nowa era w specjalnościach zabiegowych. Pol Przeg Chir 1992;64:89-93. 